\title{
Review on Region of Interest Coding Techniques for Medical Image Compression
}

\author{
Palak Jangbari \\ PG Student, Department of Electronics \& \\ Communication Engineering, \\ C.G.Patel Institute of Technology, Uka Tarsadia \\ University, Bardoli. Gujarat
}

\author{
Dhruti Patel \\ PG Student, Department of Electronics \& \\ Communication Engineering, \\ C.G.Patel Institute of Technology, Uka Tarsadia \\ University, Bardoli. Gujarat
}

\begin{abstract}
Telemedicine is a field of medicine characterized by telecommunication for clinical health care and transmission of medical images and videos. For transmission, a huge bandwidth is required over the internet. The size of the images that belongs to a single patient is very large which contains resolution factor and diagnostic images. So there is a need for efficient compression techniques for compressing these medical images. The regions which are considered to be more important than others in medical images is known as a Region of Interest (ROI) e.g. tumor is ROI in brain MRI. In this work, the ROI is detected by the saliency map technique, after that targets or ROIs be coded at available bits while the remainder of the background or non-ROI part is coded using fewer bits.By this method, the target regions within the video frame or image will be well preserved while the number of bits needed to code the video sequence or images is reduced.Thus, the transmission bandwidth and storage requirements are reduced.
\end{abstract}

\section{General Terms}

Telecommunication, Telemedicine, transmission, bandwidth

\section{Keywords}

Compression, Region of Interest (ROI), Saliency Map

\section{INTRODUCTION}

Medical images have taken an important role in diagnosis and surgery with the development of medical imaging technology. Modern and future diagnosis and surgery rely on medical images and software for practitioners, such as surgical planning, computer-aided diagnosis, simulation and robotically assisted surgical interventions. Biomedical image processing is more difficult than normal image processing because biomedical images are fuzzy in nature and with some noisy elements. For that how to process biomedical images fast and precisely is an unsolved problem and has been an important research field recently in telemedicine field. A Region of interest (ROI) is a neuropsychological concept which means the regions that people concern in the images or videos[1].Regions of interest in images often mean the important and meaningful regions in the image. The ROI is used for optimization and for ease in image processing. It can reduce computation time. There have been some methods to extract regions of interest in natural images [1], but they don't suit for medical image processing. Onsy et al. propose two methods to determine regions of interest in biomedical images [2]. One is using the mean squared error (MSE), and the other is using threshold value. But The MSE-based method requires the reference image for calculations while there is difficulty in the threshold method to determine a threshold value. In the second one, Itti's saliency map concept is used. In that different methods are used to construct the saliency map. By computing, the contrast of each pixel in the image saliency is considered. The regions of interest are extracted in the image or video frame with the help of the saliency map technique. Fig 1 shows the extraction of ROI.

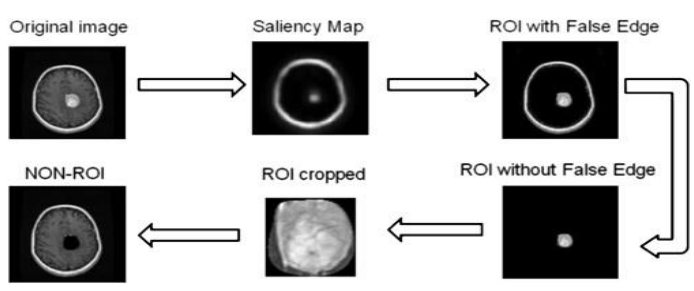

Fig 1: ROI extraction process[26]

After extraction of ROI, a compression method is used. Lossless compression is used in medical images, but the achievable compression ratio is low. The concept of Region of Interest (ROI) is considered due to some limitations of these lossy and lossless compression techniques. The loss may hamper some of diagnostically important part of the medical image. Hence, in some cases there is a need of some hybrid technique which can preserve diagnostically important part (ROI) and also provide high compression ratio [3].In such cases, these regions should be encoded with higher quality than the nonROI or background of an image. During image transmission, these regions are required to be transmitted first with higher priority [4].This whole procedure of ROI coding is shown in Fig 2 in which telecommunication of medical image is presented.

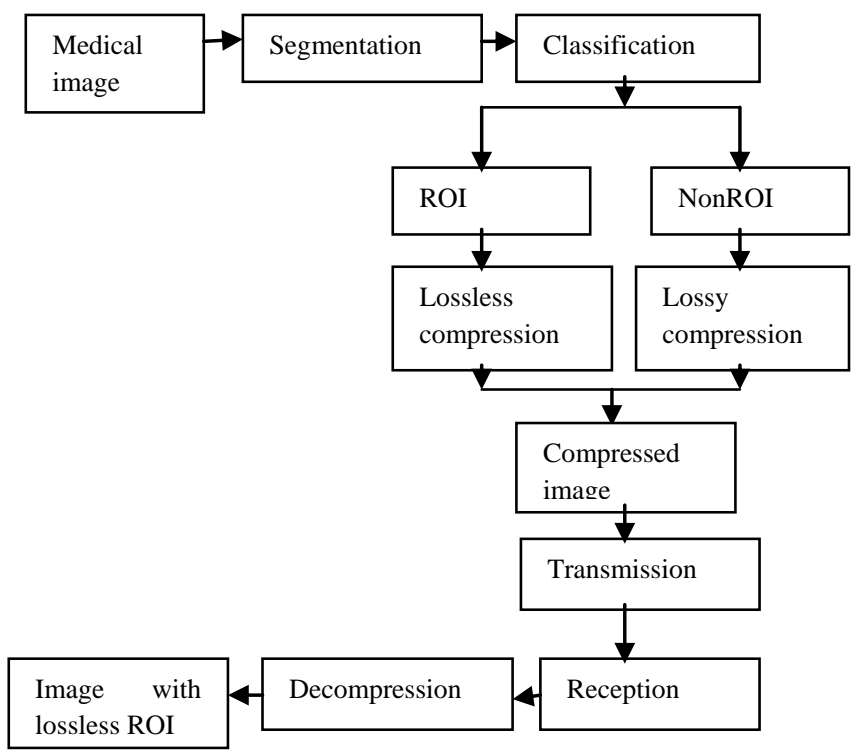

Fig 2: Block diagram of ROI coding 
Organization of this paper is organized as follow: Section 2 presents the review of related research.Section 3 presents summary of performance, problem and solution of different methods.Section 4 presents the conclusion of a paper.

\section{REVIEW OF RELATED RESEARCH}

Lossless compression of an image, its transmission and ROI coding are important aspects for a compression scheme which is used in telemedicine application.After extraction ROI is compressed with lossless compression technique such as Huffman, RLE, LZW, Arithmetic, ZIP,etc. While other than ROI part is compressed with lossy compression technique. For some factors like compression and decompression times and saving percentages of all above algorithms, the Shannon Fano algorithm is most efficient algorithm among these all lossless technique[5].The image compression ratio is in decreasing order for above algorithms as Arithmetic, Huffman, R+H, Run-Length, and LZW codings[6].

$\mathrm{ROI}$ is the important part in medical image, then one query arrise why encode the non-ROI part? Non-ROI part gives the accurate position of the ROI in an image or video frame. During the evaluation of performance of these algorithms quality measure is required for ROI and non ROI coding part.

Some other techniques are available for medical image compression.In the scaling method [7] is to scale (shift) coefficients are scaled (shifted) such that the bits required for the ROI are placed in higher bit planes than the bits required for the background. After the embedded coding, the most significant ROI bit planes are placed before the non ROI or background bit planes of the image. Some bits of the ROI coefficients may be encoded together with background coefficients according to the scaling value.The ROI will be decoded before the rest of the non ROI part of an image. Before image is fully encoded, if the encoding process is terminated or bit stream is truncated, the, the ROI will be of higher quality than the background.It is applied in nephrostogram.

In MAXSHIFT method [8], the scaling value is computed such that there is no need for transmitting shape information to the decoder and any shape of ROI can be detected.So ROI mask is not required. Depending upon the wavelet filters the mapping is done from spatial to wavelet domain.The encoder scans the quantized coefficients. It chooses a scaling value in such a way that the minimum coefficient of the ROI is larger than the maximum coefficient of the background.All of the wavelet coefficients o'f the non ROI are scaled down by ( $\mathrm{s}+$ D), where D is a small constant. The decoder scales up (by $\mathrm{s}+$ D)) all coefficients that have a magnitude $<1$.In this no shape encoding is needed. Additional coefficients are required at the decoder side to decode the region.It is also used in nephrostogram.

A lossy-to-lossless ROI compression technique is based on set partitioning in hierarchical trees (SPIHT) [10] and embedded block coding with optimal truncation (EBCOT) algorithms [11]. In this method the images are segmented into the foreground and background.For coding of shape information of ROI, chain code-based shape coding scheme [12] is used. Then critically sampled shapeadaptive integer wavelet transforms (IWTs) [13] are performed on the foreground and background image separately.Then all the foreground bit stream, the background bit stream and the shape-coding bit stream are combined and a single bit stream is created.The multipass "zerotree" coding is used to reduce the redundancy.The spatial orientation trees are divided in the wavelet domain according to whether all or some coefficients are inside or outside the ROIs [13]. The EBCOT's context model improves SPIHT's coding efficiency. After that the arithmetic coding are applied on sign bits and refinement bits. The wavelet transform used in EBCOT is replaced again with a shape-adaptive wavelet transform [17].In that a modification is made such that the coefficients inside ROIs are coded.This technique is used in the application of chromosome images. The region-based IWT (RB-IWT) [14] is also based on SPIHT [10].IWT [15] is used to obtain the representation of the partitioned image plane. There is no need of differentiation of coefficients associated with each region. It is very advantageous as low-complexity implementation is performed based on the lifting based algorithm which is also a lossless coding of given image regions. IWT maps the image which is decomposition of partition into a partitioned wavelet coefficient plane.Thus ROI is obtain. Further these arbitrary shape region's representations are encoded using a modified SPIHT scheme.This RB-IWT method performs better than SPIHT-based ROI coding according to the experimental results gven by the author.In paper [16] ROI wavelet-based coding methods are given with application to digital mammography. In those methods the region based discrete wavelet transform (RBDWT) [17] is applied, after performing a segmentation of the important region in an image.Then object-based extension of the SPIHT (OB-SPIHT) [10] coding algorithm and an object- based extension of the set partitioned embedded block (OB-SPECK) [18] coding method is used respectively for both the methods.RBDWT it is possible to performs a wavelet subband decomposition of an arbitrary shape region very efficiently.Both OB-SPIHT and OBSPECK mehods are embedded in nature,which means the method produces an embedded bit stream that can be truncated at any point at a desired quality of the image.The wavelet coefficients consist of larger information content have a larger magnitude. In a comparisonwith SPIHT and JPEG2000, OB-SPIHT and OB-SPECK gives higher quality for interested region in mammography at the same compressed file size.

The embedded zero tree wavelet (EZW) coding technique [19] is presented for ROI coding during a progressive transmission of videos or images. The frequency band of the input image is split into different subbands to reduce the correlation using subband decomposition and wavelet transform at different resolutions. In EZW the shape information is not required for coding. An arbitrary region can be compressed because of partially localized wavelet transform. In that corresponding wavelet coefficients of the ROI are given in wavelet domain and then the EZW algorithm is applied on these coefficients. Additional coefficients are needed at the decoder side to decode the object. It is applicable in knee CT scans and mammography.

Vector quantization (VQ) is another ROI coding technique [20]. In this the image is divided into small nonoverlapping blocks. Each vector of the image is compared to the elements of a codebook and only the index of the nearest code vector is transmitted. In the ROI-VQ, a separate codebook is generated for every region .The properties of these codebooks are chosen according to the medical feature. For diagnostically important part, a large codebook containing small codewords is created and for less important non ROI, the block size is smaller.This compression technique has been used for echoendoscopic images. The ROI-VQ method is used for a good rate/distortion performance and quality of ROI. 
ROI coding for volumetric medical images

Three-dimensional (3-D) wavelet coding provides better performance compared to corresponding 2-D methods.One method is SA-DWT[21], exists in which only samples within an image are transformed.It requires shape information at the decoder side which we have to additionally send. Compared to scaling-based ROI (SB-ROI), some samples are required to encode the image.For that more efficient coding is required.In user-driven ROI coding, the user specifies an ROI during the coding process. In SA-DWT[17], when the ROI is specified the wavelet transform needs to be performed again.This causes more computational cost. The ROI and background are reproduced without the pixel blending artifacts as in MAXSHIFT algorithm.SA-ROI has better compression performance compared to SB-ROI.

A hybrid model is one of the techniques of lossless compression in the ROI.In this algorithm high-rate, motioncompensation and lossy compression in other regions are applied.It is used for colon CT images.The colon wall is segmented through a 3-D morphological techniques.The output of the motion-compensated acts as an initial approximation for ROI.After the ROI is segmented, a hybrid compression is used. The first slice is compressed by the lossless coder.After that, each slice is coded by motioncompensated coding. At the end difference between the input image ROI and the predicted ROI block is coded by an entropy-minimizing lossless coder.

An extension to 3D-SPIHT [25] which allows 3-D ROI coding.In that 3-D bitmap mask is maintained at the encoder side to identify the coefficients that affect the ROI.For this mask, a wavelet transform is used.In that for the perfect reconstruction of the ROI, a necessary coefficient is identified at each step.Tthe coefficients of the bitmap mask are shifted at a fixed number of a left bit as to give greater priority to ROI. Similar to general scaling method, it is quite simple and efficient algorithm.

\section{SUMMARY OF DIFFERENT METHODS}

\section{Table 1: Performance of different methods}

\begin{tabular}{|l|l|l|}
\hline Method & Paper & Comments \\
\hline Scaling method & (7) & $\begin{array}{l}\text { For 2D still image, Arbitrary } \\
\text { roi supported, Part of } \\
\text { JEPG2000 The scaling } \\
\text { method image can be } \\
\text { resolved even in case of } \\
\text { truncated bit stream. }\end{array}$ \\
\hline MAXSHIFT & $(8)$ & $\begin{array}{l}\text { For 2D still image, } \\
\text { Additional coefficient } \\
\text { require, Arbitrary ROI } \\
\text { supported, Simple decoding } \\
\text { process without needing ROI } \\
\text { mask generation }\end{array}$ \\
\hline $\begin{array}{l}\text { SPIHT-based } \\
\text { coding }\end{array}$ & $(9)$ & $\begin{array}{l}\text { For 2D still image, Use for } \\
\text { lossy and loss less both }\end{array}$ \\
\hline $\begin{array}{l}\text { lossy-to-lossless ROI } \\
\text { compression scheme }\end{array}$ & $(10)$ & $\begin{array}{l}\text { For 2D still image ,Shape } \\
\text { information require, } \\
\text { multipass "zerotree" coding } \\
\text { is adapted in order to reduce } \\
\text { the redundancy. }\end{array}$ \\
\hline
\end{tabular}

\begin{tabular}{|l|l|l|}
\hline $\begin{array}{l}\text { Automated region- } \\
\text { based } \\
\text { compression }\end{array}$ & $(22)$ & $\begin{array}{l}\text { For 2D still image } \\
\text { Automatic Arbitrary ROI } \\
\text { supported, automated, } \\
\text { efficient and low complexity, } \\
\text { lossless, scalable }\end{array}$ \\
\hline EBCOT & $(11)$ & $\begin{array}{l}\text { For 2D still image,Arbitrary } \\
\text { ROI supported }\end{array}$ \\
\hline RB-IWT & $(14)$ & $\begin{array}{l}\text { For 2D still image ,low- } \\
\text { complexity, Arbitrary ROI } \\
\text { supported 2D still image, }\end{array}$ \\
\hline ROI-VQ & $(19)$ & $\begin{array}{l}\text { For 2D coefficient } \\
\text { Additional ROI } \\
\text { require, Arbitrary } \\
\text { supported, progressive image } \\
\text { transmission }\end{array}$ \\
\hline Extended 3D-SPIHT & $(25)$ & $\begin{array}{l}\text { For 2D still image Obtains } \\
\text { good rate/distortion } \\
\text { performance preserving ROI } \\
\text { quality }\end{array}$ \\
\hline SA-DWT & $(20)$ & $\begin{array}{l}\text { For volumetric image,More } \\
\text { efficient coding,better } \\
\text { compression } \\
\text { lossless reconstruction and }\end{array}$ \\
\hline
\end{tabular}

Table 2: Different methods problem and solution

\begin{tabular}{|c|c|c|}
\hline Method & Problem & Solution \\
\hline Scaling method & $\begin{array}{l}\text { Additional coefficient } \\
\text { require }\end{array}$ & $\begin{array}{l}\text { SPIHT,R } \\
\text { B-IWT }\end{array}$ \\
\hline MAXSHIFT & $\begin{array}{l}\text { Slightly higher bit rates, } \\
\text { additional coefficients } \\
\text { to decode the object }\end{array}$ & $\begin{array}{l}\text { Lossy- } \\
\text { lossless } \\
\text { technique }\end{array}$ \\
\hline $\begin{array}{l}\text { SPIHT-based ROI } \\
\text { coding }\end{array}$ & Complexity & MSPIHT \\
\hline $\begin{array}{l}\text { lossy-to-lossless } \\
\text { ROI compression } \\
\text { scheme }\end{array}$ & $\begin{array}{l}\text { ROI must be } \\
\text { predetermined }\end{array}$ & RB-IWT \\
\hline $\begin{array}{lr}\text { Automated } & \text { region- } \\
\text { based } & \text { hybrid } \\
\text { compression } & \end{array}$ & $\begin{array}{l}\text { Future scope ,exact } \\
\text { decoding of the object } \\
\text { not possible }\end{array}$ & - \\
\hline EBCOT & $\begin{array}{l}\text { Additional coefficient } \\
\text { require }\end{array}$ & Maxshift \\
\hline RB-IWT & $\begin{array}{l}\text { Not progressive image } \\
\text { transmission,exact } \\
\text { decoding of the object } \\
\text { not possible }\end{array}$ & SWT \\
\hline
\end{tabular}




\begin{tabular}{|l|l|l|}
\hline EZW & $\begin{array}{l}\text { constraint on the bit rate } \\
\text { is limited to the size of } \\
\text { the ROI }\end{array}$ & ROI-VQ \\
\hline ROI-VQ & $\begin{array}{l}\text { Arbitrary ROI not } \\
\text { supported }\end{array}$ & SA-DWT \\
\hline SA-DWT & $\begin{array}{l}\text { Requires more } \\
\text { computation power than } \\
\text { other techniques. }\end{array}$ & \\
\hline $\begin{array}{l}\text { Extended } \\
\text { SPIHT }\end{array}$ & 3D- & \\
\hline
\end{tabular}

\section{CONCLUSION AND FUTURE SCOPE}

This paper discusses the overview of ROI coding techniques.In this paper medical image segmentation, different ROI extraction saliency based techniques,lossy and lossless compression etc are explained.The performance of those methods are summarised in the form of table.ROI and non ROI both the parts are important for compression in medical image. ROI-based coding provides better compression as compared to other lossless methods [26]. The general scaling, the MAXSHIFT, the EZW based and the ROI-VQ methods are complex as they require additional coefficients at the decoder side, whereas in the rest of the techniques are coded using the same number of coefficients as the entire image. Among all some of those methods require the ROI shape information, whereas most of them support arbitrary ROI coding which are explained earlier.SPIHT technique gives highest PSNR among all.[29] So complexity and PSNR are to be considered for better performance during implementation.For different purposes different techniques are used.

\section{FUTURE SCOPE}

Different methods are described here.According to the application they are used.It is necessary to adapt the algorithms to decrease the complexity. This will be used in portable and mobile devices,in which limited computing power is required.It will be used in low bit rate coding as ROI coding is considered.

\section{REFERENCES}

[1] Xiaodi Hou and Liqing Zhang, "Saliency Detection: A Spectral Residual Approach," IEEE Conference on Computer Vision and Pattern Recognition, 2007, pp.1-8.

[2] Onsy Abdel Alim1, Nadder Hamdy and Wesam Gamal El-Din,"Determination of the Region of Interest in the Compression of Biomedical Images," 24th National Radio Science Conference,2007,pp.1-6.

[3] Miaou S G, Ke F S and Chen S C,"A lossless compression method for medical image sequences using JPEG-LS and interframe coding." IEEE Trans. Inform. Technol. Biomed.,2009, 13(5): 818-821.

[4] Maglogiannis I and Kormentzas G,"Wavelet-based compression with ROI coding support for mobile access to DICOM images over heterogeneous radio networks." Trans. Inform. Technol. Biomed,2009, 13(4):458-466.

[5] S.R. Kodituwakku, U. S.Amarasinghe, "Comparision of lossless datacompression algorithms for text data", ISSN : 0976-5166,2010,vol 1,pp. 416-425.
[6] D.Wu and E.C.Tan, "Comparison of lossless image compression algorithms," IEEE conference on TENCON 1999, vol.1, pp.718 - 721 .

[7] ISO/IEC JTC 1/SC 29/WG 1 (ITU-T SG8), JPEG 2000 Part II Final Committee Draft, Dec. 2000.

[8] ISO/IEC JTC 1/SC 29/WG 1 (ITU-T SG8), JPEG 2000 Part I Final Committee Draft, Version 1.0,Mar. 2000.

[9] Z. Liu, J. Ha, Z. Xiong, Q. Wu, and K. Castleman, "Lossy-to-lossless ROI coding of chromosome images using modified SPIHT and EBCOT," in Proc. IEEE Int. Symp. Biomedical Imaging, Washington, DC, 2002,p. 317.

[10] A. Said and W.A. Pearlman, "A new, fast and efficient image codec based on set partitioning in hierarchical trees," IEEE Trans. Circuits Syst. Video Technol. 1996, vol. 6 ,pp. 243-250.

[11] D. Taubman, "High performance scalable image compression with EBCOT," IEEE Trans. Image Processing,2000, vol. 9, pp. 1158-1170

[12] Z. Liu, J. Ha, Z. Xiong, Q. Wu, and K. Castleman, "Cascaded differential and wavelet compression of chromosome images," IEEE Trans. Biomed. Eng.,2002, vol. 49, pp. 323-283.

[13] G. Minami, Z. Xiong, A. Wang, and S. Mehrotra, "3-D wavelet coding of video with arbitrary regions of support," IEEE Trans. Circuits Syst. VideoTechnol., 2001,vol. 11, pp. 1063-1068.

[14] S. Tasdoken and A. Cuhadar, "ROI coding with integer wavelet transforms and unbalanced spatial orientation trees," presented at 25th Ann. Int. Conf. IEEE EMBS, Mexico, Sept. 2003.

[15] S. Dewitte and J. Cornelis, "Lossless integer wavelet transform," IEEE Signal Processing Lett.,1997, vol. 4, pp.158-160.

[16] M. Penedo, W.A. Pearlman, P.G. Tahoces, M. Souto, and J.J. Vidal,"Region-based wavelet coding methods for digital mammography," IEEE Trans. Med. Imag., Oct. 2003, vol. 22, pp.1288-1296.

[17] S. Li and W. Li, "Shape-adaptive discrete wavelet transform for arbitrary shaped visual object coding," IEEE Trans. Circuits Syst. Video Technol., Aug. 2000,vol. 10, pp. 725-743.

[18] A. Islam and W.A. Pearlman, "An embedded and efficient low-complexity hierarchical image coder," in Proc. SPIE, Dec. 1999, vol. 3653, p. 294-305.

[19] R. Dilmaghani, A. Ahmadian, M. Ghavami, M Oghabian, and H.Aghvami, "Multi rate/resolution control in progressive medical image transmission for the region of interest (ROI) using EZW," presented at the 25thAnn. Int. Conf. IEEE EMBS, Mexico, Sept. 2003.

[20] A. Cziho, G. Cazuguel, B. Solaiman, and C. Roux, "Medical imagecompression using region-of-interest vector quantization," in Proc. $20^{\text {th }}$ Ann. Int. Conf. IEEE EMBS, Hong Kong, 1998,p. 1277.

[21] I. Ueno and W. Pearlman, "Region of interest coding in volumetricimages with shape-adaptive wavelet transform," in Proc. SPIE, May 2003,vol. 5022,pp. $1048-1055$ 
[22] S.B. Gokturk, C. Tomasi, B. Girod, and C. Beaulieu, "Medical image compression based on region of interest, with application to colon CT images," in Proc. 23rd Ann. Int. Conf. IEEE EMBS, Istanbul,2001, p. 2453.

[23] Z. Xiong, X. Wu, and D.Y. Yun, "Progressive coding of medical volumetric data using three-dimensional integer wavelet packet transform," in Proc. 2nd IEEE Workshop Multimedia Signal Processing, Redondo Beach,CA, Dec. 1998, pp. 553-558.

[24] G. Bernabe, J. Gonzalez, J.M. Garcia, and J. Duato, “A new lossy 3-D wavelet transform for high-quality compression of medical video," in Proc. IEEE EMBS Int. Conf. Information Technology Applications in Biomedicine,Arlington, VA, Sept. 2000, p. 226.

[25] D. Gibson, M. Spann, and S.I. Woolley, "A waveletbased region of interest encoder for the compression of angiogram video sequences," IEEE Trans. Inform. Technol. Biomed., Jun. 2004,vol. 8, pp. 103-113.
[26] Bairagi, V.K.; Sapkal, A.M., "Automated region-based hybrid compression for digital imaging and communications in medicine magnetic resonance imaging images for telemedicine applications," in Science, Measurement \& Technology, IET ,July 2012, vol.6, no.4, pp.247-253.

[27] Lan, Xuguang, Nanning Zheng, Wen Ma, and Yuan Yuan. "Arbitrary ROI-based wavelet video coding." Neurocomputing 74, 2011,no.12,pp. 2114-2122.

[28] Bairagi, Vinayak K., and Ashok M. Sapkal. "ROI-based DICOM image compression for telemedicine." Sadhana 38,2013, no. 1,pp. 123-131.

[29] Doukas, C., Maglogiannis, I.: 'Region of interest coding techniques for medical image compression', IEEE Eng. Med. Biol. Mag., 2007, 26, (5),pp. 29-35. 